\title{
Thermal Treatment of Nylon 6 Fiber with Super Heat Steam under Atmospheric Pressure
}

\author{
Part 2: Thermal Treatment of Running Yarns \\ By Munetoshi Yamashita and Fumihide Fujimoto, Members, TMSJ \\ Katata Research Institute, Toyobo Co., Ltd., Otsu, Shiga-ken \\ Based on the Journal of the Textile Machinery Society of Japan, Transactions, Vol.24, No.4, T69-74, (1971)
}

\begin{abstract}
The previous article dealt with the fundamental behaviors of nylon 6 fibers subjected to thermal treatment with super heat steam under atmospheric pressure and compared them with those treated with dry heat.

This article concerns the effect of heat treatment with steam of running yarns (non-twisted and false twisted yarn) and compares it with the effect of treatment with dry heat.

1) The thermal effect on the density of non-twisted nylon 6 yarn shows exactly the same tendency (i.e., minimum point at about $150^{\circ} \mathrm{C}$ ) as in the previous article. In false-twisted yarn, the density gradually increases as temperature rises.

2) The surface filaments of false twisted yarn are affected by the relative humidity in the treatment chamber. Its inner filaments are influenced by the moisture which the filaments absorb before treatmen.t.

3) The crimp of yarn texturized with steam is superior in character to the crimp of yarn treated with dry heat.
\end{abstract}

KEY WORDS: NYLON 6, FILAMENTS, HEAT TREATMENT, DENSITY, DIFFUSION COEFFICIENT, MOISTURE, TEXTURED YARNS (FILAMENT), FALSE TWIST

\section{Introduction}

The previous article showed that the degree of the effect of thermal treatment of nylon 6 with super heat steam depended on humidity in the chamber at treating temperature. The present article reports on experiments in which thermal treatment with super heat steam was conducted by using a practical process (e.g., texturing of yarn). A machine for making false-twist textured yarn was used in the experiments.

\section{Experiments and Measurement}

2-1 Sample: nylon 6 70D/24f (cold drawn yarn)

2-2 Apparatus: Treatment chamber (see Fig. 1) placed in the heatsetting zone of the false-twist machine.

2-3 Treating condition: $7 \mathrm{~g} / \mathrm{min}$ of steam or air was let into the chamber. Yarn was supplied at the rate of 20 $\mathrm{m} / \mathrm{min}$ (retention time: 1.8 seconds) under $3 \%$ stretch. Yarn was conditioned and tested at $20^{\circ} \mathrm{C}, 50 \% \mathrm{RH}$. Yarn was treated at $100^{\circ}-200^{\circ} \mathrm{C}$ and twisted at 0 and $3100 \mathrm{~T} / \mathrm{m}$.

2-4 Measuring yarn properties: Density of treated yarn was measured by a gradient tube method using tetra-

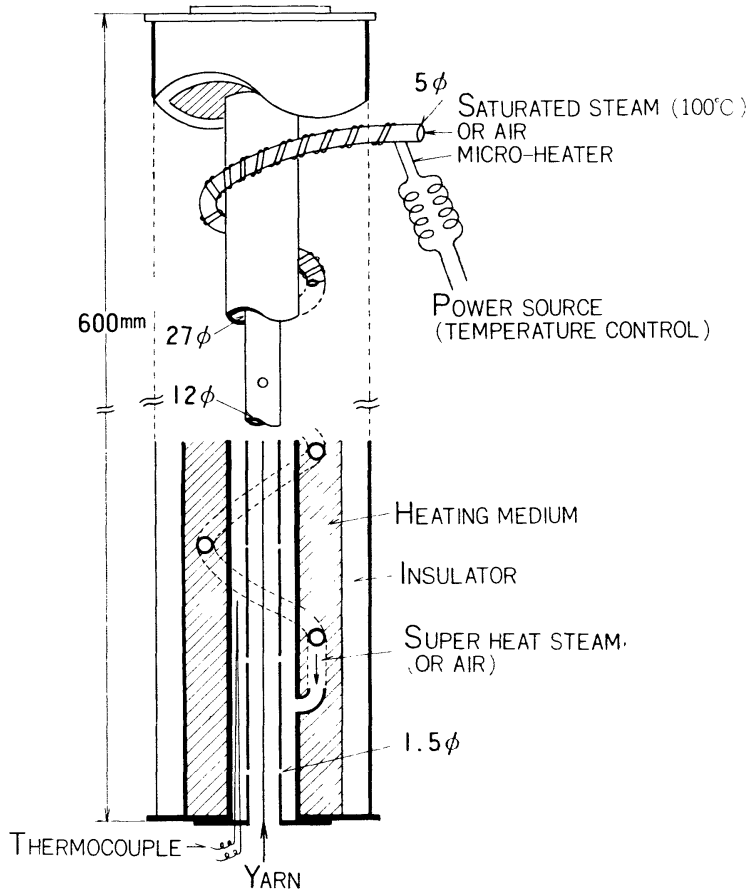

Fig. 1 Schematic illustration of heating chamber 
chloroethane and toluene at $25^{\circ} \mathrm{C}$. The properties of crimped yarn were measured mainly by the Heberlein method. ${ }^{[1]}$

To measure crimp rigidity $\left(\mathrm{CR}_{1}\right)$, crimped yarn was straightened by imposing a plumb bob $0.2 \mathrm{~g} / \mathrm{d}$ in weight on it. The length of the yarn was measured by putting a bob weighing $0.002 \mathrm{~g} / \mathrm{d}$ on it. Apparent crimp recovery $\left(\mathrm{CR}_{2}\right)$ was evaluated by using the $0.02 \mathrm{~g} / \mathrm{d}$ bob, not the $0.002 \mathrm{~g} / \mathrm{d}$. Crimp durability in boiling water $\left(\mathrm{CR}_{3}\right)$ was evaluated mainly by Harada's method. ${ }^{[2]}$ During treatment in boiling water, the yarn had a bob $0.005 \mathrm{~g} / \mathrm{d}$ in weight imposed on it.

\section{Results Discussed}

3-1 Dependence of Temperature on Density of Yarn during Thermal Treatment

Fig. 2 shows the dependence of temperature on the density of non-twisted yarn during heat treatment with super heat steam. This treatment produced the same results as in the previous article. So did dry heat treatment.

On the other hand, the relationship between the density of false-twisted yarn and the treating temperature is as shown in Fig. 3. The density curves of super heat steam and air look alike in Fig. 3. No increase in density was observed at temperatures below $150^{\circ} \mathrm{C}$, in contrast to Fig. 2. Nor was there a minimum point as in Fig. 2.

The effect on non-twisted yarn treated with super heat steam was different from that on false-twisted yarn similarly treated. The reason is presumably that, in the steam treatment of non-twisted yarn, its constituent filaments received the treatment in a uniform degree; but that, in the case of twisted yarn, its surface filaments and inner filaments received different effects from steam treatment. False-twisted yarn takes hexagonal closed packing during texturing treatment. ${ }^{[3]}$

The density values $(0$ or $\bigcirc$ ) shown in Figs. 2 and 3 were measured as multifilaments $70 \mathrm{D} / 24 \mathrm{f}$. The points shown ( and $\bigcirc$ ) represent the density of multifilaments $(70 \mathrm{D} / 24 \mathrm{f})$. That is, these values of density represent the mean value of the surface and inner filaments of twisted yarn.

\section{3-2 Density Distribution of Treated Yarn}

The density of the individual monofilaments of treated yarn was measured to explain the effect of heat treatment on surface and inner filaments.

It was very difficult to separate a surface and inner filament individually, because of the way the filaments of twisted yarn traverse in it. Therefore, treated yarn was cut in a $0.5 \mathrm{~cm}$ length and density of the cut fibers measured in a gradient tube. The distribution of the density of the treated yarn in various conditions is shown in Figs. 4-a and b. " $n$ "' is the total number of measured filaments, of which $24 \times 3$ were initially used for measuring. Then the number

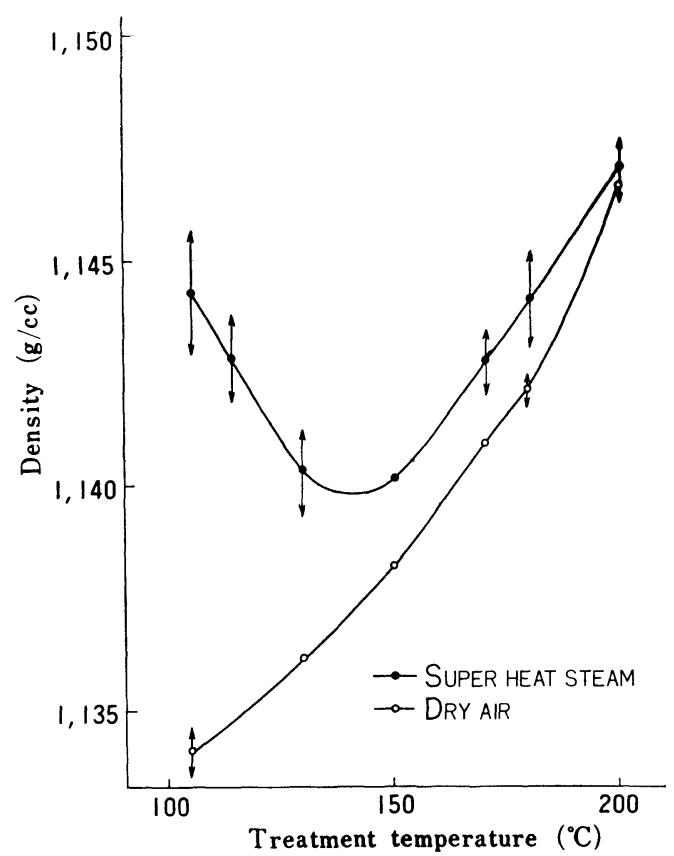

Fig. 2 Dependence of temperature on density of non twisted yarn; and distribution of density

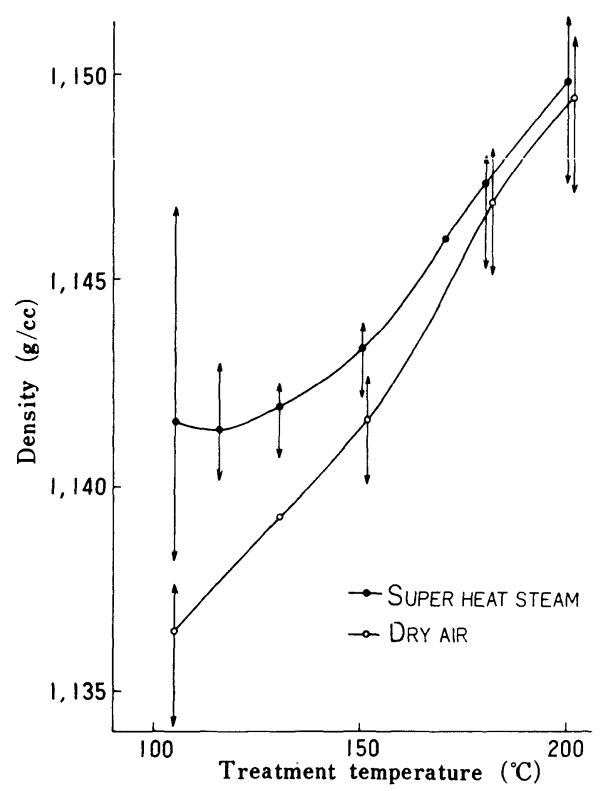

Fig. 3 Dependence of temperature on density of false twisted yarn; and distribution of density

of measured filaments was designated as $n=24$, and the width of the value of measurement was divided in one tenth. The density of false-twisted yarn treated with super heat steam at low temperatures makes a broad distribution. 
Yarn treated with dry air also makes a broader than nontwisted yarn does. Presumably, the moisture of the surface filaments vaporizes rapidly and the moisture of the inner filament scarcely vaporizes within the retention time of treatment-with the result that the surface and inner filament get different effects from treatment.

3-3 Relationship between Shrinkage and Condition of Treatment

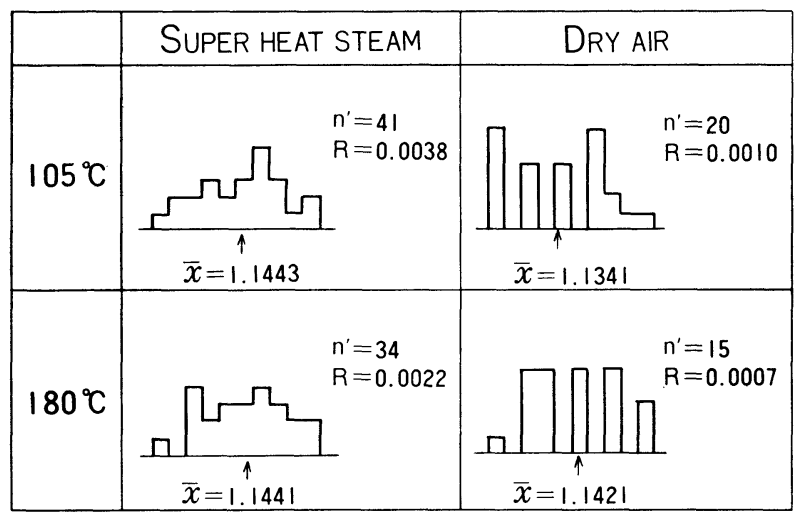

Fig. 4-a Distribution of density of false twisted yarn

(cf) n': number of monofilaments measured for density

$\mathrm{R}$ : width of distribution of density $(\mathrm{g} / \mathrm{cc})$

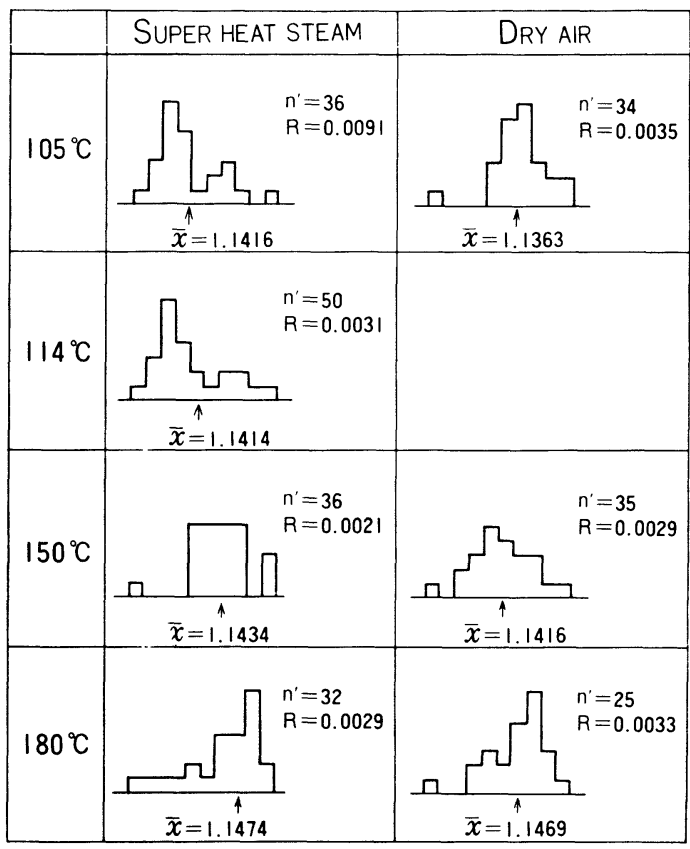

Fig. 4-b Distribution of density of non twisted yarn

(cf) n': number of nomofilaments measured for density

$\mathrm{R}$ : width of distribution of density $(\mathrm{g} / \mathrm{cc})$

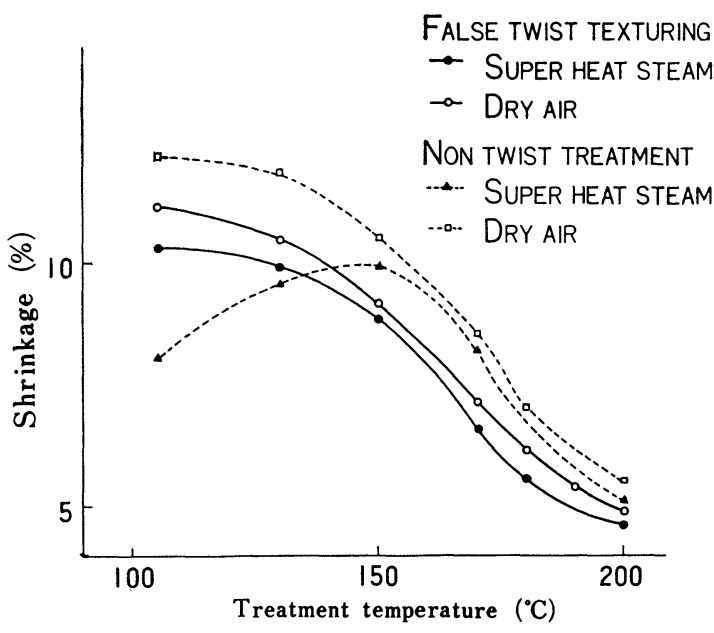

Fig. 5 Shrinkage in boiling water

Fig. 5 shows the shrinkage rates of non-twisted and false-twisted yarns treated with heat steam and those treated with dry air. The shrinkage of non-twisted yarn treated with super heat steam had a maximum point of $150^{\circ} \mathrm{C}$, described in the previous article and shown in Fig. 2 of the present article. The shrinkage of non-twisted falsetwisted yarns treated with dry air decreased as temperature rose.

3-4 Properties of Crimped Yarn

Crimp rigidity $\left(\mathrm{CR}_{1}\right)$, apparent crimp recovery $\left(\mathrm{CR}_{2}\right)$ and crimp durability in boiling water $\left(\mathrm{CR}_{3}\right)$ were measured as properties of texturized yarn. The dependency of the temperature of treatment on $\mathrm{CR}_{1}$ is as shown in Fig. 6. $\mathrm{CR}_{1}$ of texturized yarn treated with super heat steam did not change proportionally to the rise of the temperature, as compared with that of dry air. Yarn treated with super heat steam was higher in $\mathrm{CR}_{1}$ than that yarn treated with dry air.

$\mathrm{CR}_{2}$ and $\mathrm{CR}_{3}$ of yarn treated with super heat steam also exceeded those of yarn treated with dry air and are as shown in Figs. 7 and 8. There was a good relationship between $\mathrm{CR}_{3}$ and the density of texturized yarn (Fig. 9). 3-5 Difference in Treatment Effect between Surface and Inner Filaments of Twisted Yarn

The yarn may become almost the same temperature as treatment chamber during about $2 \mathrm{sec}$. of treatment, considered from report of D.F. Auther, et al. ${ }^{[4]}$ who studied on temperature of yarn of nylon during false twist texturing.

Thermal treatment of twisted yarn is complicated in its effect because its surface filaments are more sensitive to humidity in the treatment chamber than its inner filaments. This prompts us to discuss the effect of treatment of twisted yarn with super heat steam.

Nylon yarn $(70 \mathrm{D} / 24 \mathrm{f})$ has a radius of $50 \mu$ (radius of monofilaments: about $10 \mu$ ) when twisted in hexagonal 


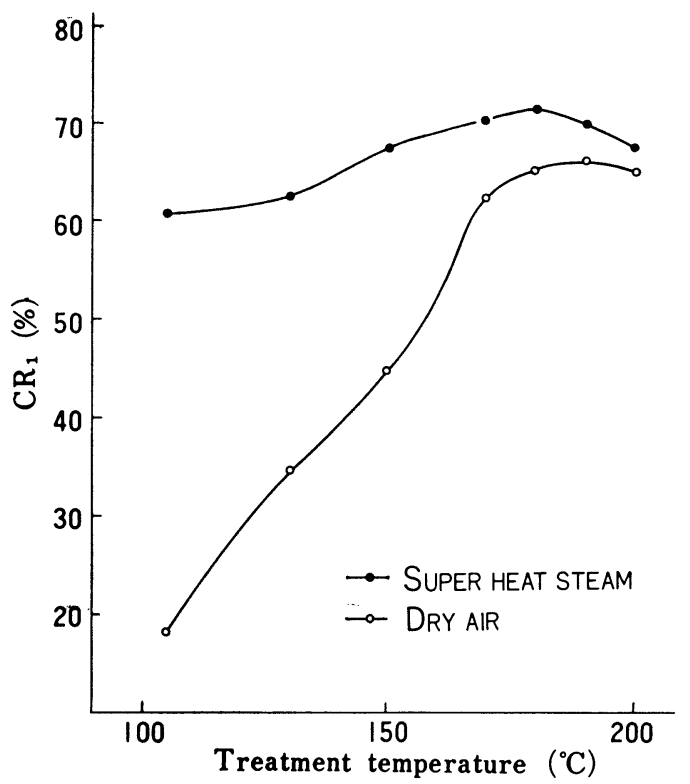

Fig. $6 \mathrm{CR}_{2}$ vs. false twisting temperature

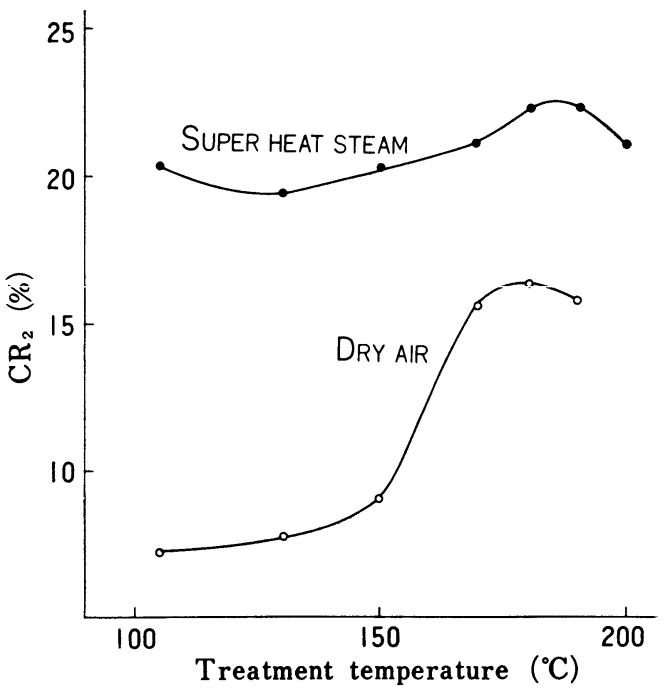

Fig. $7 \mathrm{CR}_{2}$ vs. false twisting temperature

closed packing. The diffusion coefficients of water in nylon 6 fibers are about $10^{-7} \mathrm{~cm} / \mathrm{sec}$ at $110^{\circ} \mathrm{C}$ and about $10^{-6}$ $\mathrm{cm} / \mathrm{sec}$ at $160^{\circ} \mathrm{C}$. They are values extrapolated from reported values. ${ }^{[5]}$ The distance of diffusion of water from the surface of twisted yarn during texturing was calculated by Hill's equation ${ }^{[6]}$ :

$\log \left(1-C_{t} / C_{\infty}\right)=-0.1599-2.5124 D \cdot t / r^{2}$

where $C_{\infty}$ : equilibrium amount of water absorbed

$C_{t}$ : amount of water absorbed at $t \mathrm{sec}$

$t$ : absorption time

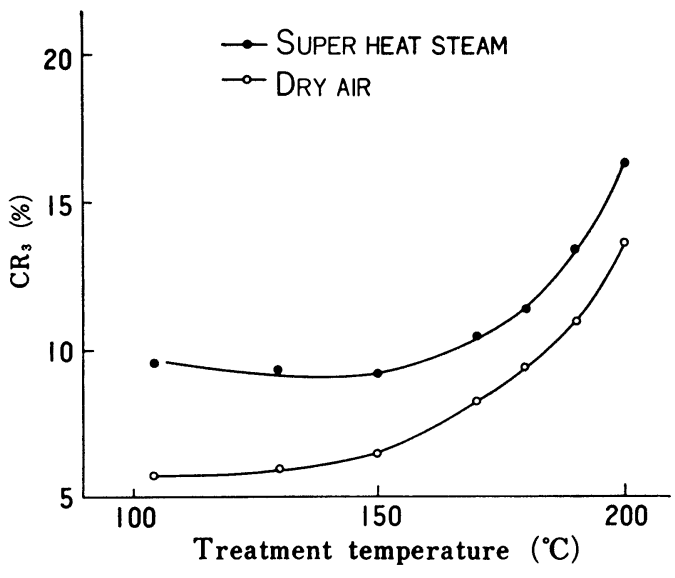

Fig. $8 \mathrm{CR}_{3}$ vs. false twisting temperature

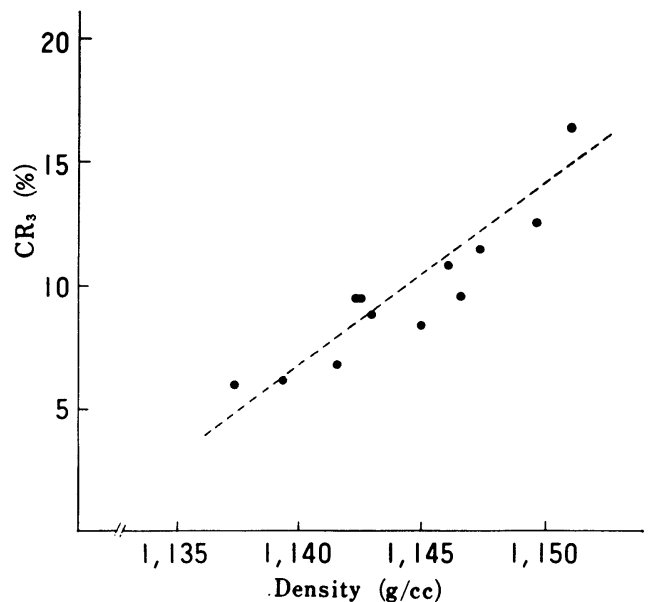

Fig. 9 Relationship between density of false twisted yarn and crimp property $\left(\mathrm{CR}_{3}\right)$

$D: \quad$ diffusion coefficient

$r$ : radius of yarn

Water can diffuse to $9 \mu$ from the yarn surface at $110^{\circ} \mathrm{C}$ and $17 \mu$ at $160^{\circ} \mathrm{C}$ in 2 seconds. Presumably, then, only the surface filaments of twisted yarn are affected by humidity in the chamber. When yarn is texturized with super heat steam at $120^{\circ} \mathrm{C}$, the surface filaments may be affected by the steam, but the inner filaments have almost the same effect as by treatment with dry air. It appears from Fig. 2 that the surface filaments have a higher density than the inner.

There may be a relationship between the distribution of density and the number of surface filaments. The number of surface filaments of false twisted yarn having hexagonal closed packing was calculated in the case of symmetrical configurations of 24-filament yarn, and the configulations are illustrated in Figs. 10-a and b. The arrow shows a shift of filaments. 


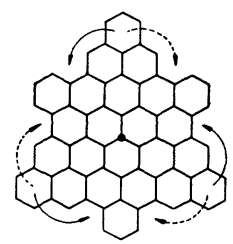

(a)

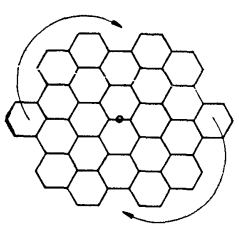

(b)

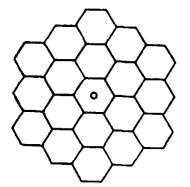

(c)

Fig.10 Schematic illustration of hexagonal closed packing of 24 filaments in symmetrical position

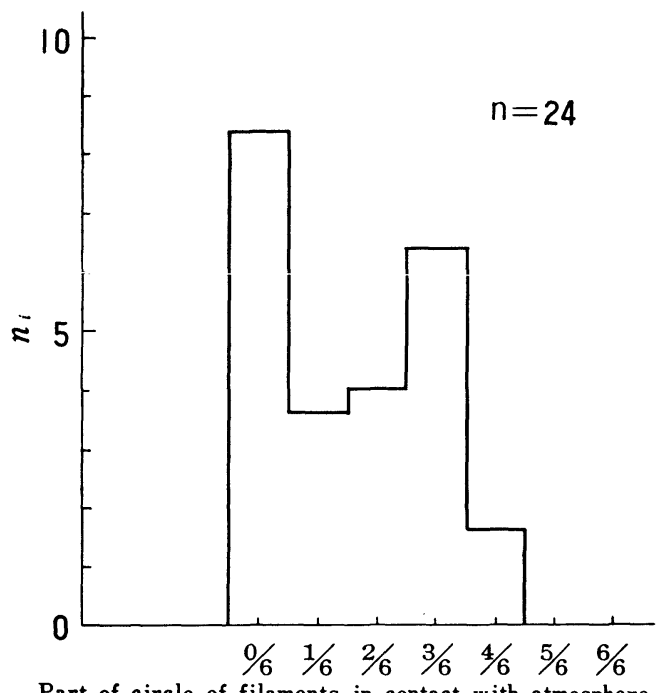

Part of circle of filaments in contact with atmosphere

Fig. 11 Distribution of surface in contact with atmosphere

The five cases obtained from Figs. $10 \mathrm{a}$ and $\mathrm{b}$ were investigated by dividing surface of each monofilament into six parts, the surface area being represented thus:

a) Surface circle of filament in contact with atomsphere ...6/6

b) Surface of filament partially in contact with atmosphere ...5/6, 4/6, 3/6, 2/6, 1/6

c) Surface of filament not in contact with atmosphere $\ldots 0 / 6$

Vol. 17 No.s 5/6 (197I)
Fig. 11 gives the mean distribution obtained from five cofigurations. The distribution shown in Fig. 11 is similar to that of the density of yarn treated with super heat steam at $105^{\circ}$ and $114^{\circ} \mathrm{C}$.

The density of surface filaments was higher than that of inner filaments, both treated with super heat steam at a low temperature (e.g. $110^{\circ} \mathrm{C}$ ). But the density of surface filaments was lower than that of the inner filaments when treatment was at $150^{\circ}$ or $180^{\circ} \mathrm{C}$, and the distribution of density was also different from that treated at a low temperature.

There was a distribution of denstiy even if false-twisted yarn was treated with dry air, because moisture in surface filaments evaporated more quickly than that in inner filaments. The density curve of surface filaments in relation to the treatment temperature (Fig. 12) is presumably the same as dry treatment shown in Fig. 2. On the other hand, inner filaments are presumably affected the same as in steam treatment, because it is difficult for moisture in the inner filaments to evaporate.

In dry treatment, the density of surface filaments may be lower than that of inner filaments. In treatment with super heat steam, surface filaments absorb moisture from the atmosphere at a low temperature (e.g. $50 \% \mathrm{RH}$ at $120^{\circ} \mathrm{C}$ ), but it desorbs moisture at a high temperature because the atmosphere has relatively low humidity (e.g., moisture regain of fibers is about $0.5-0.8 \%$ in $10 \% \mathrm{RH}$ at $180^{\circ} \mathrm{C}$ ). Thus, surface filaments treated with super heat steam at a high temperature are almost the same in behavior as those treated with dry air. These facts are illustrated in Fig. 12.

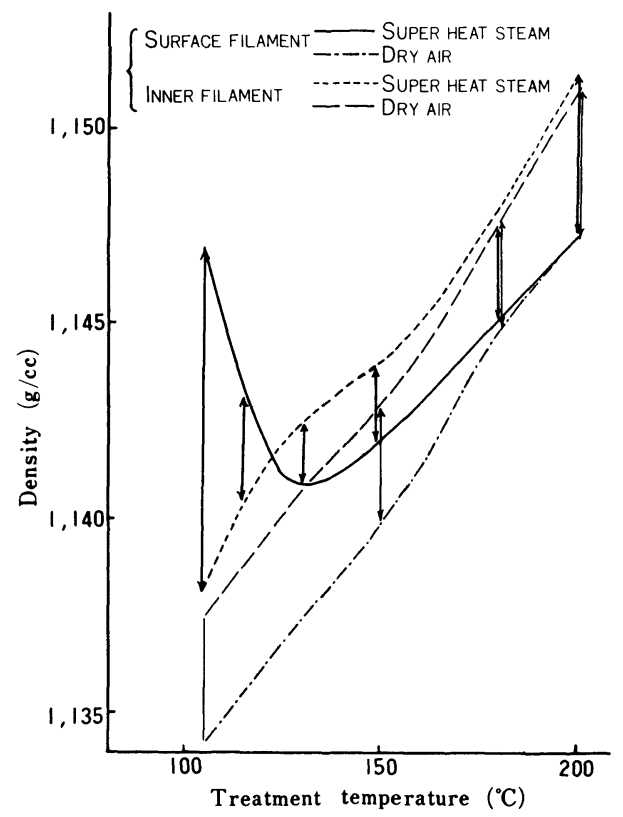

Fig. 12 Effect of thermo-setting on surface filaments and inner filaments of false twisted yarn 


\section{Conclusions}

The effect of treatment of running nylon 6 yarn with super heat steam has been studied and compared with the effect of treatment with dry air. The results obtained:

1) The density of non-twisted yarn treated with super heat steam is much higher than that treated with dry air at a low temperature (up to about $150^{\circ} \mathrm{C}$ ).

This tendency is the same as described in the previous article.

2) The density of the surface filaments of false-twisted yarn treated with steam is different from that of its inner filaments. The surface filaments are affected by the relative humidity in the treatment chamber; the inner filaments, by the moisture regain previously contained in them. 3) The properties of crimped yarn treated with super heat steam were better than those of crimped yarn treated with dry air.

\section{References}

[1] K. Kawasaki; Kakoshi-gairon, p.165 (1968) The Textile Machinery Society of Japan.

[2] T. Harada; J. Text. Mach. Soc. Japan, 22, T97(1969)

[3] N. Biegler; Melliand Textiber., 50, 85 (1969/1)

[4] D. F. Auther, C. R. Jones; J. Text. Inst., 53, T217 (1962)

[5] F. H. Müller, E. Hellmuth; Kolloid Z. 177, 1 (1961)

[6] Y. Kawaguchi; private letter 